Bundesgesundheitsbl

https://doi.org/10.1007/s00103-020-03095-x

(c) Springer-Verlag GmbH Deutschland, ein

Teil von Springer Nature 2020, korrigierte

Publikation 2020

Bekanntmachung des Umweltbundesamtes

\title{
Umgang und Gebrauch von additiven Fertigungsverfahren (,3D-Druckern“) in Privathaushalten
}

\section{Stellungnahme der Innenraumlufthygiene- Kommission}

\section{Was sind 3D-Drucker und welche Einsatzgebiete gibt es für zu Hause?}

Unter dem Begriff „3D-Druck“ sind hier die additiven Fertigungsverfahren gemeint, die entweder Kunststofffilamente (FFF, Fused Filament Fabrication) oder Harze (SLA, Stereolithografie) als Werkstoffe verarbeiten und für den nicht professionellen Anwender zugänglich sind.

FFF ist aufgrund der einfachen Handhabung und des stetig sinkenden Preises für Drucker und Materialien das dabei am häufigsten verwendete Verfahren.

Beim FFF-Verfahren wird ein thermoplastischer Kunststoff (Filament) in einem Druckkopf geschmolzen. Der zähflüssige Kunststoffstrang wird durch die zweidimensionale Bewegung des Druckkopfes und einer absenkbaren Objektplatte so positioniert, dass durch lagenweisen Aufbau ein dreidimensionales Objekt entsteht. Die Größe dieser „Desktop“-Geräte ähnelt herkömmlichen Tischdruckern.

Anfangs vor allem von Modellbauern und Hobbybastlern genutzt, wird der 3DDruck immer beliebter, um beispielsweise Spielzeuge, Ersatzteile oder Haushaltsgegenstände herzustellen. Zahlreiche digitale Modelle sind online verfügbar oder können selbst im 3D-Zeichenprogramm entworfen werden.

Vor allem der stetig sinkende Preis (Einsteigermodelle gibt es bereits ab $200 €)$ sowie die zunehmende Vermarktung als „Spielzeugfabrik“ lassen die Ab- satzzahlen kontinuierlich steigen. Zudem ist der 3D-Druck in Bildungseinrichtungen in zunehmendem Maße präsent.

Mittlerweile ist eine Vielzahl verschiedener Materialien verfügbar. Fast alle FFF-Desktop-3D-Drucker können mit Acrylnitril-Butadien-Styrol (ABS) oder Polylactid (PLA) als Filament betrieben werden. Andere Filamente wie Polyethylenterephthalat (PET) werden für die 3D-Drucktechnik zu Hause seltener eingesetzt. Wichtig, sowohl für die Funktion als auch für eventuelle Freisetzungen von Stoffen beim Drucken, sind auch die den Kunststoffen zugesetzten Additive, die Materialien wie Metall, Holz, Karbonfasern und Kohlenstoff-Nanoröhrchen (engl. Carbonanotubes, CNT) einschlieBen.

\section{Welche Auswirkungen haben 3D-FFF-Drucker auf die Raumluftqualität?}

Während der Erwärmung des Kunststoffes im Druckkopf werden gasförmige Stoffe und Partikel an die Raumluft abgegeben. Diese Emission hängt maßgeblich von der Drucktemperatur und dem verwendeten Kunststoff ab [1, 2].

3D-Drucker für den Hausgebrauch sind nicht luftdicht verschlossen und verfügen über keine Absaugung, weshalb freigesetzte Stoffe beinahe ungehindert in die Raumluft gelangen können. Einige Geräte verwenden Abdeckhauben oder sind mit zusätzlichen Luftfiltern zum Zu- rückhalten von ultrafeinen Partikeln ausgestattet, deren Wirksamkeit jedoch nicht allgemein belegt ist. Zudem werden damit keine gasförmigen Stoffe zurückgehalten [3].

\section{Welche Folgen kann der 3D-Druck für die menschliche Gesundheit haben?}

Grundsätzlich unterliegen 3D-Tischdrucker für den Hausgebrauch keinen spezifischen Sicherheitsanforderungen in Bezug auf die beim Drucken freigesetzten Gase und Partikel.

Informationen bezüglich Herkunft und genauer Zusammensetzung der Inhaltsstoffe von Filamenten, die meist über das Internet bestellt werden, sind für den Verbraucher häufig nur eingeschränkt verfügbar.

Die beim FFF-Druck freigesetzten Gase und Partikel können Stoffe wie Weichmacher, Flammschutzmittel, Monomere, Antioxidantien, feste Additive, metallische Komponenten u.v.m. sowie flüchtige organische Verbindungen (volatile organic compounds $=$ VOC) enthalten [4-8]. Die Mengen sind einerseits vom verwendeten Kunststoff und dessen Drucktemperatur, andererseits von der Größe des Druckobjekts abhängig.

Besonders ABS-Kunststoffe wiesen bei Messungen höhere Stofffreisetzungen auf [9]. Die Größenverteilung der emittierten Partikel lag überwiegend im Nanometerbereich. Es handelt sich damit um alve- 
olengängige Partikeln, die bis in kleinste Verzweigungen der Lunge eindringen und in den Blutkreislauf übertreten können [11]. Bei Untersuchungen der Bundesanstalt für Materialforschung (BAM) lag die freigesetzte Partikelanzahl bei $10^{11}$ bis $10^{13}$ Partikel pro Gramm Filamenteinsatz [4].

Dass beim Drucken Stoffe freigesetzt werden, kann schon an der mitunter starken Geruchsentwicklung bemerkt werden. Über die gemessenen, gasförmig freigesetzten Stoffe ist bekannt, dass sie zu Reizerscheinungen im Atemtrakt und an den Augen führen können [10]. In einer Studie über kurzzeitige Exposition gesunder Probanden konnten zwar bisher keine klinisch relevanten Effekte beobachtet werden, dennoch wird weiter untersucht, inwiefern sensible Personengruppe wie Asthmatiker und Allergiker beim 3D-Druck durch mögliche entzündliche Reaktionen im Atemwegsbereich gefährdet sein könnten [12]. Hinweise darauf, dass neben den Atemwegen auch das Herz-Kreislaufsystem auf Freisetzungen aus 3D-Druckern reagieren könnte, ergeben sich aus einem Experiment mit Nagetieren, bei dem sich Veränderungen des Blutdrucks und der Funktion kleiner Blutgefäße zeigten [13].

Auch Stoffaufnahmen über die Haut oder - gerade bei Kinderspielzeugen oder Gegenständen, die mit Lebensmitteln in Kontakt kommen - über eine orale Aufnahme sind per se nicht auszuschließen [14].

Kommerziell hergestellte Gegenstände sind oft aus den gleichen Kunststoffen gefertigt wie bei 3D-Druckern zuhause. Ein gewerblicher Hersteller unterliegt allerdings der Verpflichtung, dafür zu sorgen, dass sein Produkt den geltenden Sicherheitsanforderungen genügt. Beim 3DDruck für den Eigenbedarf ist dies nicht der Fall. So können zuhause auch Gegenstände und Spielzeuge mittels 3D-Druck hergestellt werden, die geltende Sicherheitsanforderungen nicht erfüllen, so dass ihre technische und gesundheitliche Sicherheit möglicherweise nicht gegeben ist.

\section{Was sollte man beachten, wenn man zuhause einen 3D-Drucker nutzen möchte?}

1. Lüften: Es sollte für eine ausreichende Belüftung gesorgt werden. Da der Druckprozess mitunter mehre- re Stunden dauern kann, empfiehlt es sich, bei häufigerem Geräteeinsatz den Drucker in einem extra Raum zu betreiben, der nicht ständig betreten werden muss und separat belüftet wird.

2. Geräteumhausung: Einige Desktop-3D-Drucker werden mit (mehr oder weniger) geschlossenen Gehäusen angeboten. Von den Herstellern sollte in der Bedienungsanleitung klar dokumentiert sein, wie das Gehäuse beim Drucken zu verwenden bzw. zu verschließen ist. Studien, mit denen die Wirksamkeit bezüglich der Verringerung von Stoffeinträgen in den Innenraum durch solche Umhausungen verlässlich nachgewiesen wurden, liegen der IRK derzeit nicht vor.

3. Abstand vom Gerät halten: Um sich nicht unnötig möglichen Emissionen auszusetzen, sollte ein längeres Sitzen direkt vor dem Drucker möglichst vermieden werden. Das Fortschreiten des Druckprozesses sollte, sofern notwendig, nur von Zeit zu Zeit kurz überprüft werden.

4. Herstellerangaben beachten: Die Angaben der Hersteller von Drucker und Filament zur richtigen Düsentemperatur (der Extrusionstemperatur) sowie anderen einzustellenden Parametern beim Drucker sollten beachtet werden. Temperaturabweichungen können zu erhöhter Stofffreisetzung führen.

\section{Professionelle und kommerziell be-}

triebene 3D-Drucker sind nicht für den Hausgebrauch geeignet und dürfen ohnehin nur unter Beachtung von Arbeitsschutzauflagen betrieben werden. Privat hergestellte Druckerzeugnisse und Utensilien sollen nicht im Rahmen einer Geschäftstätigkeit an Dritte weitergegeben oder veräußert werden.

6. 3D-Drucker sind kein Kinderspielzeug und sollen von Kindern deshalb nie ohne Aufsicht betrieben werden.

Die IRK empfiehlt, die Gerätentwicklung bei 3D-Druckgeräten für den Hausgebrauch analog der bei herkömmlichen zweidimensionalen Druckern dahingehend voranzutreiben, dass durch Prüfungen der Emissionen und Prüfzeugnis- vergabe, z. B. über den Blauen Engel, die Entwicklung besonders emissionsarmer Geräte und Materialien forciert wird.

\section{Literatur}

1. Gu J, Wensing M, Uhde E, Salthammer T (2019) Characterization of particulate and gaseous pollutants emitted during operation of a desktop 3D printer. Environ Int 123:476-485

2. Stabile L, Scungio M, Buonanno G, Arpino F, Ficco $G$ (2017) Airborne particle emission of a commercial 3D printer: the effect of filament material and printing temperature. Indoor Air 27:398-408

3. Gu J, Uhde $E$, Wensing $M$, Xia F, Salthammer T (2019) Emission control of desktop 3D printing: the effects of a filter cover and an air purifier. Environ Sci Technol Lett 6:499-503

4. Seeger S, Brodner D, Jacobi T, Rasch F, Rothardt M, Wilke $O$ (2018) Emissions of fine and ultrafine particles and volatile organic compounds from different filament materials operated on a low-cost 3D printer. Gefahrstoffe - Reinhaltung Luft 3:79-87

5. Floyd EL, Wang J, Regens JL (2017) Fume emissions from a low-cost 3-D printer with various filaments. J Occup Environ Hyg 14:523-533

6. Zontek TL, Ogle BR, Jankovic JT, Hollenbeck SM (2017) An exposure assessment of desktop 3D printing. J Chem Health Saf 24:15-25

7. Kim Y, Yoon C, Ham S, Park J, Kim S, Kwon O, Tsai PJ (2015) Emissions of nanoparticles and gaseous material from 3D printer operation. Environ Sci Technol 49:12044-12053

8. Davis AY, Zhang Q, Wong JPS, Weber RJ, Black MS (2019) Characterization of volatile organic compound emissions from consumer level material extrusion 3D printers. Build Environ 160:106209

9. Azimi P, Zhao D, Pouzet C, Crain NE, Stephens B (2016) Emissions of ultrafine particles and volatile organic compounds from commercially available desktop three-dimensional printers with multiple filaments. Environ Sci Technol 50:1260-1268

10. Sagunski H (1998) Richtwerte für die Innenraumluft: Styrol. Bundesgesundhbl 41:392-398

11. Ad-hoc-Arbeitsgruppe Innenraumrichtwerte. (2008) Gesundheitliche Bedeutung von Feinstaub in der Innenraumluft. Bundesgesundhbl 51:1370-1378

12. Gümperlein I, Fischer E, Dietrich-Gümperlein $G$, Karrasch S, Nowak D, Jörres RA, Schierl R (2018) Acute health effects of desktop 3D printing (fused deposition modeling) using acrylonitrile butadiene styrene and polylactic acid materials: An experimental exposure study in human volunteers. Indoor Air 00:1-13

13. Stefaniak AB, LeBouf RF, Duling MG, Yi J, Abukabda $A B$, McBride CR, Nurkiewicz TR (2017) Inhalation exposure to three-dimensional printer emissions stimulates acute hypertension and microvascular dysfunction. Toxicol Appl Pharmacol 335:1-5

14. Oskui SM, Diamante G, Liao C, Shi W, Gan J, Schlenk D, Grover WH (2016) Assessing and reducing the toxicity of 3D-printed parts. Environ Sci Technol Lett 3:1-6 